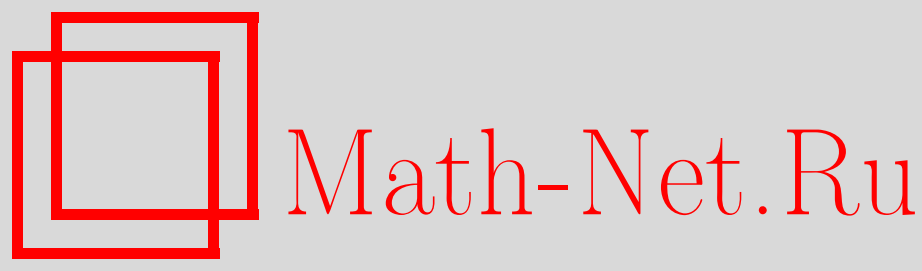

А. В. Латышев, А. А. Юшканов, Задача Смолуховского в металле с зеркально-диффузными граничными условиями, ТМФ, 2009, том 161, номер 1, 95-108

DOI: https://doi.org/10.4213/tmf6422

Использование Общероссийского математического портала Math-Net.Ru подразумевает, что вы прочитали и согласны с пользовательским соглашением http://www . mathnet.ru/rus/agreement

Параметры загрузки:

IP: 3.93 .64 .190

26 апреля 2023 г., 18:12:30

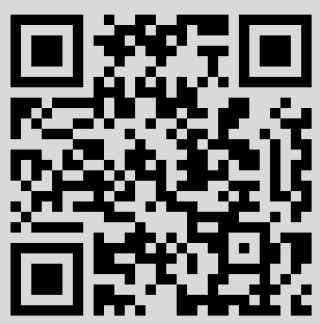




\title{
ФИЗИКА
}

Том 161, № 1

октябрь, 2009

2009 г.

\author{
А. В. Латышев*, А. А. Юшканов*
}

\section{ЗАДАЧА СМОЛУХОВСКОГО В МЕТАЛЛЕ \\ С ЗЕРКАЛЬНО-ДИФФУЗНЫМИ ГРАНИЧНЫМИ УСЛОВИЯМИ}

\begin{abstract}
Решена задача Смолуховского о распределении температуры электронного газа вблизи поверхности металла при наличии нормального к поверхности градиента температуры. Рассеяние электронов на границе металла предполагалось зеркально-диффузным. Развит специальный метод решения, использующий ряды Неймана.
\end{abstract}

Ключевые слова: металл, электронный газ, распределение температуры, градиент температуры, коэффициент зеркальности.

\section{1. ВВЕДЕНИЕ}

Задача о температурном скачке электронного газа в металле рассматривалась в наших работах [1], [2]. В этих работах получено аналитическое решение задачи о скачке температуры с учетом характера аккомодации энергии электронов на поверхности. Скачок температуры обусловлен потоком тепла к поверхности. Выведены формулы для скачка температуры и разности потенциалов электрического поля, построены профили распределения температуры и электрического потенциала в полупространстве металла.

Настоящая работа является продолжением работ [1], [2]. Здесь получено решение задачи о распределении температуры электронов в металле, когда электроны рассеиваются на поверхности зеркально-диффузно. Коэффициент зеркальности предполагается произвольным. Для решения этой задачи развивается специальный математический аппарат, суть которого заключается во введении граничных условий в кинетическое уравнение с помощью члена типа источника.

Задача о скачке температуры является одной из важнейших в кинетической теории [3]. Аналитическое решение этой задачи для случая одноатомного газа получено в работе [4], а для молекулярных газов - в работе [5]. Эта задача актуальна для случая металла, в частности, при низких температурах, когда длина свободного пробега электронов велика. Большой интерес вызывает вопрос о распределении

* Московский государственный областной университет, Москва, Россия. E-mail: avlatyshev@mail.ru,yushkanov@inbox.ru 


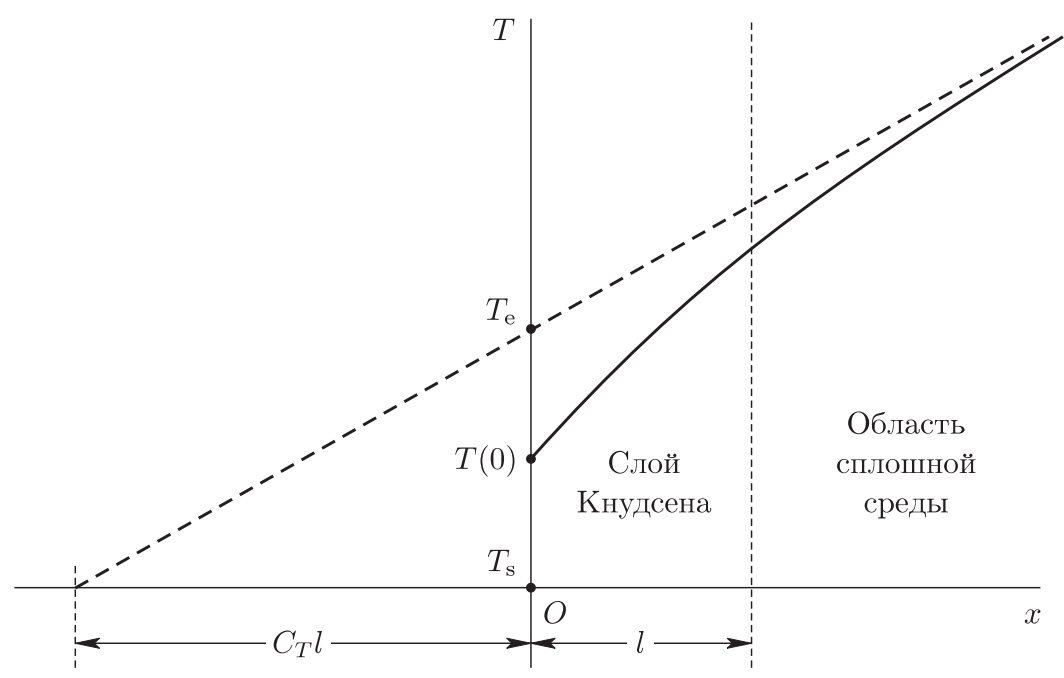

Рис. 1. Распределение температуры газа над плоской стенкой.

температуры вблизи поверхности металла, особенно для металлических образцов малого размера. Этот случай является типичным в микроэлектронике. Существенное влияние на тепловые процессы вблизи поверхности оказывает коэффициент зеркальности отражения электронов от границы металла.

Несмотря на большую теоретическую и практическую значимость вопроса о распределении температуры вблизи поверхности металла, длительное время отсутствовало аналитическое решение соответствующей задачи для случая зеркально-диффузного граничного условия. Цель данной работы - устранить этот пробел.

Рассмотрим плоскую границу раздела между металлом и окружающей средой. Предположим, что имеется поток тепла в металле, текущий к (или от) поверхности. Введем декартову систему координат с центром на поверхности и осью $x$, перпендикулярной поверхности и направленной вглубь металла. Тогда на расстояниях от поверхности, существенно превышающих длину свободного пробега электронов $l$, потоку тепла соответствует постоянный градиент температуры $G_{T}=d T / d x$ (здесь и далее будем пренебрегать возможной анизотропией свойств металла).

Слой, примыкающий к границе раздела и имеющий толщину порядка $l$, будем называть, как это принято в кинетической теории [3], слоем Кнудсена. Вне слоя Кнудсена (см. рис. 1) профиль температуры имеет вид $T(x)=T_{\mathrm{e}}+G_{T} x$, где $T_{\mathrm{e}}-$ экстраполированная температура. Обозначим температуру поверхности через $T_{\mathrm{s}}$, а истинную температуру газа непосредственно у стенки через T(0). Скачком температуры называют величину $\Delta T=T_{\mathrm{e}}-T_{\mathrm{s}}$. Эта величина при малых градиентах температуры пропорциональна градиенту $G_{T}: \Delta T=C_{T} l G_{T}$. Коэффициент $C_{T}$, не зависящий от $G_{T}$, называется коэффициентом скачка температуры. Для определения коэффициента $C_{T}$ необходимо решить кинетическое уравнение вблизи поверхности металла в слое Кнудсена. Вместо абсолютного скачка температуры $\Delta T$ обычно 
рассматривают относительный скачок $\varepsilon_{T}=C_{T} l g_{T}, g_{T}=G_{T} / T_{\mathrm{s}}$ - логарифмический градиент температуры.

Отметим, что на величину скачка температуры существенное влияние оказывает характер взаимодействия электронов с поверхностью металла. Это обстоятельство требует модификации граничных условий с целью учета коэффициента зеркальности отражения электронов на границе. Подобный учет проводится посредством введения соответствующего коэффициента зеркальности для описания отражения электронов от поверхности и привлечения общей зеркально-диффузной схемы взаимодействия электронов с поверхностью металла. Такие модифицированные условия называются граничными условиями Максвелла.

Целью данной работы является нахождение скачка температуры и распределения температуры в непосредственной близости от поверхности металла. Данное вычисление проводится путем решения кинетического уравнения для электронов с учетом коэффициента зеркальности отражения электронов на границе. Отметим, что мы рассматриваем общий случай произвольной степени вырождения электронного газа, поэтому полученные результаты справедливы в широком диапазоне температур и для широкого класса материалов.

\section{2. КИНЕТИЧЕСКОЕ УРАВНЕНИЕ}

Рассмотрим металл, в котором ферми-поверхность имеет сферическую форму. В кинетике металлов часто используется кинетическое уравнение для электронов в так называемом $\tau$-приближении [6], [7]:

$$
\frac{\partial f}{\partial t}+(\mathbf{v} \nabla) f+e_{0} \mathbf{E} \frac{\partial f}{\partial \mathbf{p}}=\frac{1}{\tau}\left(f_{\mathrm{F}}^{0}-f\right),
$$

где $f$ - функция распределения электронов, $e_{0}$ - заряд электрона, $\mathbf{p}$ - импульс электрона, $\mathbf{E}$ - электрическое поле, $\mathbf{v}$ - скорость электронов, $\tau$ - время релаксации электронов, $f_{\mathrm{F}}^{0}$ - фермиевская функция распределения.

В равновесном состоянии металла плотность заряда электронов нейтрализуется равной по величине, но противоположной по знаку плотностью заряда ионов. В связи с этим уравнение Максвелла для электрического поля в металле можно записать в виде

$$
\operatorname{div} \mathbf{E}=e_{0} \int\left(f-f_{\mathrm{F}}^{\mathrm{s}}\right) d \Omega_{\mathrm{F}}, \quad d \Omega_{\mathrm{F}}=\frac{2 d^{3} p}{(2 \pi \hbar)^{3}},
$$

где $\hbar$ - постоянная Планка, $f_{\mathrm{F}}^{\mathrm{s}}$ - фермиевская функция распределения с параметрами поверхности.

Для нейтрального ферми-газа аналогичное (1) уравнение рассматривалось в работе [8]. При конечной температуре необходимо учитывать, что время релаксации $\tau$ может зависеть от скорости движения электронов. Для полностью вырожденного электронного газа это непринципиально, так как в кинетическом процессе участвуют только электроны на ферми-поверхности с одинаковой скоростью (скоростью Ферми). При конечной температуре это уже не так, и вопрос о зависимости времени релаксации от скорости электронов приобретает существенное значение.

4 Теоретическая и математическая физика, т. 161, № 1, 2009 г. 
Если доминирует рассеяние электронов на примесях, то наиболее разумным представляется предположение о постоянстве длины свободного пробега электронов. При этом время релаксации $\tau$ будет пропорционально скорости электронов, т.е. $\tau=l / w$. Через $l$ мы обозначили длину свободного пробега электронов, а $w=|\mathbf{v}-\mathbf{u}|$, где $\mathbf{u}$ - средняя скорость движения электронов. Отметим, что в металлах во всех физически реализуемых случаях выполняется условие $u \ll v$. Поэтому в дальнейшем везде вместо $w$ будем писать $v$. Следовательно, частота столкновений электронов: $\nu(v)=1 / \tau(v)=v / l$.

При конечной температуре в уравнении (1) в качестве равновесной функции распределения электронов вместо функции $f_{\mathrm{F}}^{0}$, соответствующей нулевой температуре, должна присутствовать фермиевская функция распределения с некоторой эффективной температурой $T_{*}$ и эффективным химическим потенциалом $\mu_{*}$. Отметим, что указанные величины не совпадают, вообе говоря, с локально-равновесными значениями температуры и химического потенциала.

Тогда кинетическое уравнение для электронов в $\tau$-приближении запишется (см. [1], [2]) в следующем виде:

$$
\frac{\partial f}{\partial t}+(\mathbf{v} \nabla) f+e_{0} \mathbf{E} \frac{\partial f}{\partial \mathbf{p}}=\frac{v}{l}\left(f_{\mathrm{F}}^{*}-f\right),
$$

где $f_{\mathrm{F}}^{*}-$ функция распределения Ферми (фермиан), $f_{\mathrm{F}}^{*}=f_{\mathrm{F}}\left(\mu_{*}, T_{*}\right)$,

$$
f_{\mathrm{F}}\left(\mu_{*}, T_{*}\right)=\left[\exp \left(\frac{m v^{2}}{2 k_{\mathrm{B}} T_{*}}-\frac{\mu_{*}}{k_{\mathrm{B}} T_{*}}\right)+1\right]^{-1},
$$

$k_{\mathrm{B}}$ - постоянная Больцмана, $m$ - эффективная масса электрона.

Закон сохранения числа электронов приводит к равенству, следующему из уравнения (3):

$$
\int v f d \Omega_{\mathrm{F}}=\int v f_{\mathrm{F}}^{*} d \Omega_{\mathrm{F}} .
$$

Из кинетического уравнения (3) для электронов в стационарном случае вытекает соотношение

$$
\nabla \mathbf{Q}_{e}=\frac{1}{l}\left(\int v \frac{m}{2} v^{2} f_{\mathrm{F}}^{*} d \Omega_{\mathrm{F}}-\int v \frac{m}{2} v^{2} f d \Omega_{\mathrm{F}}\right),
$$

где $\mathbf{Q}_{e}$ - поток тепла, переносимый электронами.

В стационарном случае при отсутствии источников тепла полный поток тепла $\mathbf{Q}$ сохраняется, т.е. $\nabla \mathbf{Q}=0$. Если вкладом фононов в перенос тепла в металле можно пренебречь, то и $\nabla \mathbf{Q}_{e}=0$, а из (5) следует, что

$$
\int v \frac{m}{2} v^{2} f d \Omega_{\mathrm{F}}=\int v \frac{m}{2} v^{2} f_{\mathrm{F}}^{*} d \Omega_{\mathrm{F}} .
$$

Соотношения (4) и (6) определяют параметры $T_{*}, \mu_{*}$ кинетического уравнения (3). Отметим, что в рассматриваемом приближении кинетическое уравнение для электронов (3) оказывается независимым от уравнения для фононов. При этом ряд тонких эффектов, например увлечение электронов фононами, оказываются неучтенными. Для их учета, вообще говоря, необходим выход за пределы $\tau$-приближения. 
Будем считать, что массовая скорость много меньше скорости электронов, а возникающие характерные перепады температуры на длине $l$ малы по сравнению с температурой электронного газа. В этих предположениях возможна линеаризация задачи. Будем искать функцию распределения в виде

$$
f=f_{\mathrm{F}}^{\mathrm{s}}+\varphi(x, \mu, c) g,
$$

где $f_{\mathrm{F}}^{\mathrm{s}}=f_{\mathrm{F}}\left(\mu_{\mathrm{s}}, T_{\mathrm{s}}\right), \varphi-$ новая неизвестная функция, $\mu_{\mathrm{s}}-$ химический потенциал электронов, рассеянных поверхностью, $T_{\mathrm{s}}$ - температура поверхности, $c$ - модуль безразмерной скорости $\mathbf{c}=\sqrt{\beta} \mathbf{v}, \beta=m / 2 k_{\mathrm{B}} T_{\mathrm{s}}$, функция $g$ есть производная фермиана $f_{\mathrm{F}}^{\mathrm{s}}$ по безразмерному химическому потенциалу $\alpha=\mu_{\mathrm{s}} / k_{\mathrm{B}} T_{\mathrm{s}}$,

$$
f_{\mathrm{F}}^{\mathrm{s}}=\frac{1}{e^{c^{2}-\alpha}+1}, \quad g=\frac{e^{c^{2}-\alpha}}{\left[e^{c^{2}-\alpha}+1\right]^{2}} .
$$

Введем безразмерные величины

$$
\mathbf{e}=\frac{e_{0} l}{k_{\mathrm{B}} T_{\mathrm{S}}} \mathbf{E}, \quad t_{*}=\frac{t}{l \sqrt{\beta}}, \quad \mathbf{r}_{*}=\frac{\mathbf{r}}{l} .
$$

Далее звездочки у этих величин будем опускать.

Система уравнений (3) и (2), описывающих данную задачу, состоит (см. [1], [2]) из кинетического уравнения для электронов и уравнения для электрического поля:

$$
\begin{aligned}
\mu \frac{\partial \varphi}{\partial x}+\varphi(x, \mu, c)-\mu e(x) & =\frac{1}{g_{1}(\alpha)} \int_{-1}^{1} \int_{0}^{\infty} k\left(c, c^{\prime}\right) \varphi\left(x, \mu^{\prime}, c^{\prime}\right) g\left(c^{\prime}\right) c^{\prime 3} d \mu^{\prime} d c^{\prime}, \\
e^{\prime}(x) & =a_{0}^{2} \int_{-1}^{1} \int_{0}^{\infty} \varphi(x, \mu, c) g(c) c^{2} d \mu d c,
\end{aligned}
$$

где $\mu=c_{x} / c, a_{0}^{2}=e_{0}^{2} m^{2} l^{2} \sqrt{\beta} / \pi^{2} \varepsilon_{0} \hbar^{3}, \varepsilon_{0}$ - диэлектрическая постоянная,

$$
\begin{gathered}
k\left(c, c^{\prime}\right)=1+\frac{g_{1}^{2}(\alpha)}{\Delta(\alpha)}\left(c^{2}-\frac{g_{3}(\alpha)}{g_{1}(\alpha)}\right)\left(c^{\prime 2}-\frac{g_{3}(\alpha)}{g_{1}(\alpha)}\right), \\
g_{n}(\alpha)=2 \int_{0}^{\infty} g(c) c^{n} d c, \quad n=0,1,2, \ldots, \quad \Delta(\alpha)=g_{1}(\alpha) g_{5}(\alpha)-g_{3}^{2}(\alpha), \\
g_{3}(\alpha)=4 \int_{0}^{\infty} c \ln \left(1+e^{\alpha-c^{2}}\right) d c, \quad g_{1}(\alpha)=\ln \left(1+e^{\alpha}\right) \\
g_{5}(\alpha)=12 \int_{0}^{\infty} c^{3} \ln \left(1+e^{\alpha-c^{2}}\right) d c .
\end{gathered}
$$

\section{3. ГРАНИЧНЫЕ УСЛОВИЯ И ПОСТАНОВКА ЗАДАЧИ}

Пусть полупространство $x>0$ заполнено металлом, плоскость $y z$ совмещена с его границей.

Предположим, что существует поток тепла в металле, перпендикулярный поверхности. Тогда и электрическое поле, связанное с потоком тепла, будет перпендикулярно поверхности. При этом все параметры задачи будут зависеть только от 
координаты $x$. Зеркально-диффузная схема отражения электронов от поверхности означает, что функция распределения электронов $f(x, \mathbf{v})$ удовлетворяет на границе металла следующему условию:

$$
f\left(+0, v_{x}, v_{y}, v_{z}\right)=A_{0} g\left(v_{x}\right)+(1-q) f_{\mathrm{F}}^{\mathrm{s}}(v)+q f\left(+0,-v_{x}, v_{y}, v_{z}\right), \quad v_{x}>0 .
$$

Здесь $q, 0<q \leqslant 1,-$ так называемый коэффициент зеркальности, т.е. доля электронов, отражающихся от границы зеркально, $1-q$ - доля электронов, отражающихся от границы диффузно, т.е. с фермиевской функцией распределения по скоростям, $A_{0}$ - константа, учитывающая непроницаемость поверхности металла для электронов (см. [9]).

Зеркально-диффузное условие для функции $\varphi$ упрощается:

$$
\varphi\left(0, c_{x}, c_{y}, c_{z}\right)=q \varphi\left(0,-c_{x}, c_{y}, c_{z}\right)+A_{0}, \quad c_{x}>0
$$

а условие в объеме металла имеет вид

$$
\varphi(x, \mu, c)=\varphi_{\mathrm{as}}^{+}(x, \mu, c)+o(1), \quad x \rightarrow+\infty .
$$

Граничные условия для поля следующие:

$$
e(0)=0, \quad e(x)=e_{\mathrm{as}}^{+}+o(1), \quad x \rightarrow+\infty .
$$

Здесь

$$
\begin{gathered}
\varphi_{\mathrm{as}}^{+}=\left[\varepsilon_{T}+g_{T}^{+}(x-\mu)\right]\left(c^{2}-\frac{r_{0}(\alpha)}{s(\alpha)}\right)+b(\alpha) g_{T}^{+} \mu, \\
g_{T}^{+}=\left.\left(\frac{d \ln T}{d x}\right)\right|_{x=+\infty}, \\
e_{\mathrm{as}}^{+}=g_{T}^{+} b(\alpha), \quad b(\alpha)=\frac{g_{3}(\alpha)}{g_{1}(\alpha)}-\frac{g_{2}(\alpha)}{g_{0}(\alpha)}, \\
g_{2}(\alpha)=\frac{3}{2} \int_{0}^{\infty} \ln \left(1+e^{\alpha-c^{2}}\right) d c, \quad g_{0}(\alpha)=\int_{0}^{\infty} \frac{e^{\alpha-c^{2}}}{1+e^{\alpha-c^{2}}} d c .
\end{gathered}
$$

Функция $\varphi_{\text {as }}$ описывает теплопроводность электронного газа в объеме металла. Процесс теплопроводности сопровождается появлением электрического поля $e_{\text {as }}$ (термоэлектрический эффект, описанный, например, в [6]). Условие $e(0)=0$ связано с предположением об отсутствии электрического поля вне металла.

Задача состоит в нахождении такого решения системы (8), которое удовлетворяет граничным условиям (9)-(11). Подчеркнем, что функции $\varphi_{\mathrm{as}}, e_{\mathrm{as}}$ являются решением системы уравнений (8).

Согласно структуре функции $\varphi_{\text {as }}$ ищем функцию $\varphi$ в виде

$$
\varphi(x, \mu, c)=h_{1}(x, \mu)+\left(c^{2}-\frac{g_{2}(\alpha)}{g_{0}(\alpha)}\right) h_{2}(x, \mu) .
$$


Получим две задачи: задачу о температурном скачке

$$
\begin{gathered}
\mu \frac{\partial h_{2}}{\partial x}+h_{2}(x, \mu)=\frac{1}{2} \int_{-1}^{1} h_{2}(x, \mu) d \mu, \\
h_{2}(0, \mu)=q h_{2}(0,-\mu), \quad 0<\mu<1, \\
h_{2}(x, \mu)=\varepsilon_{T}+g_{T}^{+}(x-\mu)+o(1), \quad x \rightarrow+\infty, \quad-1<\mu<0,
\end{gathered}
$$

и задачу о построении электрического поля

$$
\begin{gathered}
\mu \frac{\partial h_{1}}{\partial x}+h_{1}(x, \mu)=\frac{1}{2} \int_{-1}^{1} h_{1}(x, \mu) d \mu+\mu e(x), \\
e^{\prime}(x)=\frac{a^{2}}{2} \int_{-1}^{1} h_{1}(x, \mu) d \mu, \quad a=a(\alpha)=a_{0} \sqrt{g_{0}(\alpha)}, \\
h_{1}(+0, \mu)=A_{0}+q h_{1}(+0,-\mu), \quad 0<\mu<1, \quad e(0)=0, \\
h_{1}(x, \mu)=b(\alpha) \mu g_{T}^{+}+o(1), \quad e(x)=e_{\text {as }}^{+}+o(1), \quad x \rightarrow+\infty .
\end{gathered}
$$

Далее рассмотрим задачу о температурном скачке. Разовьем метод, в котором граничное условие включается в уравнение. Этот момент требует достаточно тонких рассуждений. А именно, чтобы включить граничное условие на функцию $h_{2}$ в уравнение, продолжим сформулированную задачу на “отрицательное" полупространство $x<0$ следующим образом:

$$
h_{2}(x, \mu)=h_{2}(-x,-\mu) .
$$

Выделим у $h_{2}$ асимптотическую часть (см. формулу (15)), полагая

$$
h_{2}(x, \mu)=\varepsilon_{T}+g_{T}^{+}(x-\mu)+h_{2 c}(x, \mu) .
$$

Получаем граничные условия

$$
\begin{aligned}
h_{2 c}(+0, \mu) & =-(1-q) \varepsilon_{T}+(1+q) g_{T}^{+} \mu+q h_{2 c}(+0,-\mu), \\
h_{2 c}(+\infty, \mu) & =0, \quad 0<\mu<1 .
\end{aligned}
$$

В полупространстве $x<0$ получаем следующие граничные условия:

$$
\begin{aligned}
h_{2 c}(-0, \mu) & =-(1-q) \varepsilon_{T}-(1+q) g_{T}^{+} \mu+q h_{2 c}(-0,-\mu), \\
h_{2 c}(-\infty, \mu) & =0, \quad-1<\mu<0 .
\end{aligned}
$$

Соответствующие граничные условия для положительного и отрицательного полупространств объединим в одно. Тогда получаем

$$
\begin{gathered}
h_{2 c}( \pm 0, \mu)=-(1-q) \varepsilon_{T} \pm(1+q) g_{T}^{+} \mu+q h_{2 c}( \pm 0,-\mu), \quad 0<|\mu|<1, \\
h_{2 c}( \pm \infty, \mu)=0 .
\end{gathered}
$$




\section{4. ЗАДАЧА О РАСПРЕДЕЛЕНИИ ТЕМПЕРАТУРЫ}

Граничные условия (18) включим в кинетическое уравнение (13). Предварительно перепишем условие (18) с учетом (17):

$$
h_{2 c}( \pm 0, \mu)=-(1-q) \varepsilon_{T} \pm(1+q) g_{T}^{+} b(\alpha) \mu+q h_{2 c}(\mp 0, \mu) .
$$

Возьмем уравнение, включающее граничное условие (19):

$$
\mu \frac{\partial h_{2 c}}{\partial x}+h_{2 c}(x, \mu)=U(x)+|\mu|\left[h_{0}^{ \pm}(\mu)-(1-q) h_{2 c}(\mp 0, \mu)\right] \delta(x),
$$

где

$$
\begin{gathered}
U(x)=\frac{1}{2} \int_{-1}^{1} h_{2 c}\left(x, \mu^{\prime}\right) d \mu^{\prime}, \\
h_{0}^{ \pm}(\mu)=-(1-q) \varepsilon_{T}+(1+q) g_{T}^{+} b(\alpha)|\mu|, \quad 0<|\mu|<1 .
\end{gathered}
$$

Будем искать решения уравнений (20) и (21) в виде интегралов Фурье:

$$
\begin{gathered}
h_{2 c}(x, \mu)=\frac{1}{2 \pi} \int_{-\infty}^{\infty} e^{i k x} \Phi(k, \mu) d k \\
U(x)=\frac{1}{2 \pi} \int_{-\infty}^{\infty} e^{i k x} E(k) d k, \quad \delta(x)=\frac{1}{2 \pi} \int_{-\infty}^{\infty} e^{i k x} d k .
\end{gathered}
$$

Из соотношений $(21),(22)$ и первого из (23) находим, что

$$
E(k)=\frac{1}{2} \int_{-1}^{1} \Phi(k, \mu) d \mu .
$$

Для нахождения значений $h_{2 c}(\mp 0, \mu), 0<|\mu|<1$, поступим следующим образом. Будем решать уравнение $(20)$, считая функцию $U(x)$ известной. При этом в случае $x>0, \mu<0$ получаем

$$
h_{2 c}^{+}(x, \mu)=-\frac{1}{\mu} e^{-x / \mu} \int_{x}^{+\infty} e^{t / \mu} U(t) d t
$$

а в случае $x<0, \mu>0$ находим

$$
h_{2 c}^{-}(x, \mu)=\frac{1}{\mu} e^{-x / \mu} \int_{-\infty}^{x} e^{t / \mu} U(t) d t .
$$

Решения (25) и (26) удовлетворяют условию обращения в нуль вдали от стенки.

Теперь уравнение (20) можно переписать в виде

$$
\mu \frac{\partial h_{2 c}}{\partial x}+h_{2 c}(x, \mu)=U(x)+|\mu|\left[h_{0}^{ \pm}(\mu)-(1-q) h_{2 c}^{ \pm}(0, \mu)\right] \delta(x) .
$$


Подставим в выражение (25) первый из интегралов Фурье (23), получаем

$$
h_{2 c}^{+}(x, \mu)=-\frac{1}{\mu} e^{-x / \mu} \int_{x}^{+\infty} e^{t / \mu} \frac{d t}{2 \pi} \int_{-\infty}^{+\infty} e^{i k t} E(k) d k=\frac{1}{2 \pi} \int_{-\infty}^{+\infty} \frac{e^{i k x} E(k) d k}{1+i k \mu} .
$$

Аналогично получаем

$$
h_{2 c}^{-}(x, \mu)=\frac{1}{2 \pi} \int_{-\infty}^{+\infty} \frac{e^{i k x} E(k) d k}{1+i k \mu} .
$$

Используя четность функции $E(k)$, из двух последних равенств получаем

$$
h_{2 c}^{ \pm}(0, \mu)=\frac{1}{2 \pi} \int_{-\infty}^{+\infty} \frac{E(k) d k}{1+i k \mu}=\frac{1}{\pi} \int_{0}^{+\infty} \frac{E\left(k_{1}\right) d k_{1}}{1+k_{1}^{2} \mu^{2}} .
$$

Перейдем в уравнении (27) к интегралам Фурье, используя предыдущее равенство:

$$
(1+i k \mu) \Phi(k, \mu)=E(k)+|\mu|\left[h_{0}^{ \pm}(\mu)-(1-q) \frac{1}{\pi} \int_{0}^{+\infty} \frac{E\left(k_{1}\right) d k_{1}}{1+k_{1}^{2} \mu^{2}}\right] .
$$

Выразим функцию $\Phi(k, \mu)$ из уравнения $(28)$ и подставим ее в $(24)$. Получаем характеристическое интегральное уравнение

$$
E(k) L(k)=-(1-q) \varepsilon_{T} T_{1}(k)+(1+q) g_{T}^{+} T_{2}(k)-\frac{1-q}{\pi} \int_{0}^{\infty} J_{1}\left(k, k_{1}\right) E\left(k_{1}\right) d k_{1},
$$

где

$$
\begin{gathered}
L(k)=1-T_{0}(k)=k^{2} T_{2}(k), \quad T_{n}(k)=\int_{0}^{1} \frac{\mu^{n} d \mu}{1+k^{2} \mu^{2}}, \quad n=0,1,2, \ldots, \\
J_{n}\left(k, k_{1}\right)=\int_{0}^{1} \frac{\mu^{n} d \mu}{\left(1+k^{2} \mu^{2}\right)\left(1+k_{1}^{2} \mu^{2}\right)}, \quad n=1,2, \ldots
\end{gathered}
$$

\section{5. РЕШЕНИЕ ХАРАКТЕРИСТИЧЕСКОГО УРАВНЕНИЯ}

Будем искать решение уравнения (29) в виде рядов

$$
\begin{aligned}
E(k) & =(1+q) g_{T}^{+}\left[E_{0}(k)+(1-q) E_{1}(k)+(1-q)^{2} E_{2}(k)+\cdots\right], \\
\varepsilon_{T} & =g_{T}^{+} C_{T}(q), \quad C_{T}(q)=\frac{1+q}{1-q}\left[\varepsilon_{0}+(1-q) \varepsilon_{1}+(1-q)^{2} \varepsilon_{2}+\cdots\right] .
\end{aligned}
$$

Получаем счетное множество уравнений:

$$
\begin{aligned}
& E_{0}(k) L(k)=-\varepsilon_{0} T_{1}(k)+T_{2}(k), \\
& E_{n}(k) L(k)=-\varepsilon_{n} T_{1}(k)-\frac{1}{\pi} \int_{0}^{+\infty} J\left(k, k_{1}\right) E\left(k_{1}\right) d k_{1}, \quad n=1,2, \ldots
\end{aligned}
$$


Из уравнения (32) находим

$$
E_{0}(k)=\frac{-\varepsilon_{0} T_{1}(k)+T_{2}(k)}{L(k)} .
$$

В точке $k=0$ это выражение имеет полюс второго порядка. Для его устранения преобразуем $E_{0}(k)$ к виду

$$
E_{0}(k)=\frac{2-3 \varepsilon_{0}}{6 k^{2} T_{2}(k)}+\frac{\varepsilon_{0} T_{3}(k)-T_{4}(k)}{T_{2}(k)} .
$$

Отсюда видно, что для устранения полюса в нуле следует взять $\varepsilon_{0}=2 / 3$, тогда

$$
E_{0}(k)=\frac{\varphi_{0}(k)}{T_{2}(k)}, \quad \varphi_{0}(k)=\frac{2}{3} T_{3}(k)-T_{4}(k)
$$

Теперь из уравнения (33) при $n=1$ получаем

$$
E_{1}(k)=\frac{1}{L(k)}\left[-\varepsilon_{1} T_{1}(k)-\frac{1}{\pi} \int_{0}^{\infty} J\left(k, k_{1}\right) E_{0}\left(k_{1}\right) d k_{1}\right] .
$$

Правая часть (34) также имеет полюс второго порядка в нуле. Для его устранения разложим числитель правой части (34) по степеням $k$ :

$$
\begin{aligned}
& \varepsilon_{1} T_{1}(k)+\frac{1}{\pi} \int_{0}^{\infty} J\left(k, k_{1}\right) E_{0}\left(k_{1}\right) d k_{1}= \\
& \quad=\frac{\varepsilon_{1}}{2}+\frac{1}{\pi} \int_{0}^{\infty} T_{1}\left(k_{1}\right) E_{0}\left(k_{1}\right) d k_{1}-k^{2}\left[\varepsilon_{1} T_{3}(k)+\frac{1}{\pi} \int_{0}^{\infty} J_{3}\left(k, k_{1}\right) E_{0}\left(k_{1}\right) d k_{1}\right] .
\end{aligned}
$$

Приравнивая нулю свободный член этого разложения, находим

$$
\varepsilon_{1}=-\frac{2}{\pi} \int_{0}^{\infty} T_{1}\left(k_{1}\right) E_{0}\left(k_{1}\right) d k_{1}=0.04615 .
$$

С учетом (35) преобразуем (34):

$$
E_{1}(k)=\frac{1}{T_{2}(k)}\left[\varepsilon_{1} T_{3}(k)+\frac{1}{\pi} \int_{0}^{\infty} J_{3}\left(k, k_{1}\right) E_{0}\left(k_{1}\right) d k_{1}\right] .
$$

Для разложения интеграла $J\left(k, k_{1}\right)$ мы воспользовались равенством $J\left(k, k_{1}\right)=T_{1}\left(k_{1}\right)$ - $J_{3}\left(k, k_{1}\right)$. Подставим $(35)$ в $(36)$ и обозначим

$$
M\left(k, k_{1}\right)=J_{3}\left(k, k_{1}\right)-2 T_{3}(k) T_{1}\left(k_{1}\right),
$$

тогда имеем

$$
E_{1}(k)=\frac{1}{\pi} \int_{0}^{\infty} \frac{M\left(k, k_{1}\right)}{T_{2}(k)} E_{0}\left(k_{1}\right) d k_{1}
$$


Рассмотрим уравнение (33) при $n=2$. Аналогично рассуждая, получаем

$$
\begin{aligned}
\varepsilon_{2} & =-\frac{2}{\pi} \int_{0}^{\infty} T_{1}\left(k_{1}\right) E_{1}\left(k_{1}\right) d k_{1}=-2.512 \cdot 10^{-3}, \\
E_{2}(k) & =\frac{1}{\pi} \int_{0}^{\infty} \frac{M\left(k, k_{1}\right)}{T_{2}(k)} E_{1}\left(k_{1}\right) d k_{1} .
\end{aligned}
$$

В $n$-м приближении $(n=1,2, \ldots)$ получаем

$$
\begin{aligned}
\varepsilon_{n} & =-\frac{2}{\pi} \int_{0}^{\infty} T_{1}\left(k_{1}\right) E_{n-1}\left(k_{1}\right) d k_{1}, \\
E_{n}(k) & =\frac{1}{\pi} \int_{0}^{\infty} \frac{M\left(k, k_{1}\right)}{T_{2}(k)} E_{n-1}\left(k_{1}\right) d k_{1} .
\end{aligned}
$$

Перепишем последние два выражения через нулевое приближение с помощью кратных интегралов:

$$
\begin{aligned}
\varepsilon_{n} & =-\frac{2}{\pi^{n}} \int_{0}^{\infty} \ldots \int_{0}^{\infty} \frac{T_{1}\left(k_{1}\right) M\left(k_{1}, k_{2}\right) \ldots M\left(k_{n-1}, k_{n}\right)}{T_{2}\left(k_{1}\right) \ldots T_{2}\left(k_{n}\right)} \varphi_{0}\left(k_{n}\right) d k_{1} \ldots d k_{n}, \\
E_{n}(k) & =\frac{1}{\pi^{n}} \int_{0}^{\infty} \ldots \int_{0}^{\infty} \frac{M\left(k, k_{1}\right) M\left(k_{1}, k_{2}\right) \ldots M\left(k_{n-1}, k_{n}\right)}{T_{2}(k) T_{2}\left(k_{1}\right) \ldots T_{2}\left(k_{n}\right)} \varphi_{0}\left(k_{n}\right) d k_{1} \ldots d k_{n} .
\end{aligned}
$$

Таким образом, все члены рядов (30) и (31) построены. Выражение для скачка температуры во втором приближении имеет вид $\varepsilon_{T}(q)=C_{T}(q) g_{T}^{+}$, где $C_{T}(q)-$ коэффициент скачка температуры,

$$
C_{T}(q)=0.66667 \frac{1+q}{1-q}+0.04615(1+q)-0.00251\left(1-q^{2}\right) .
$$

\section{6. РАСПРЕДЕЛЕНИЕ ТЕМПЕРАТУРЫ}

Для построения профиля распределения температуры в полупространстве металла воспользуемся законами сохранения числа частиц и энергии:

$$
\int f d \Omega_{\mathrm{F}}=\int f_{\mathrm{F}} d \Omega_{\mathrm{F}}, \quad \int v^{2} f d \Omega_{\mathrm{F}}=\int v^{2} f_{\mathrm{F}} d \Omega_{\mathrm{F}} .
$$

Из равенств (38) получаем следующую систему уравнений для построения профилей химического потенциала и температуры:

$$
\int\left[\delta \alpha+\left(c^{2}-\alpha\right) \frac{\delta T}{T_{\mathrm{s}}}-\varphi\right] c^{n} g(c) d^{3} c=0, \quad n=0,2 .
$$

Из этой системы находим профиль распределения температуры:

$$
\frac{\delta T(x)}{T_{\mathrm{S}}}=\frac{g_{0}(\alpha)}{8 \pi \Delta_{0}(\alpha)} \int \varphi(x, \mu, c) c^{2} g(c) d^{3} c-\frac{g_{2}(\alpha)}{8 \pi \Delta_{0}(\alpha)} \int \varphi(x, \mu, c) g(c) d^{3} c,
$$

где

$$
\Delta_{0}(\alpha)=g_{0}(\alpha) g_{4}(\alpha)-g_{2}^{2}(\alpha)
$$




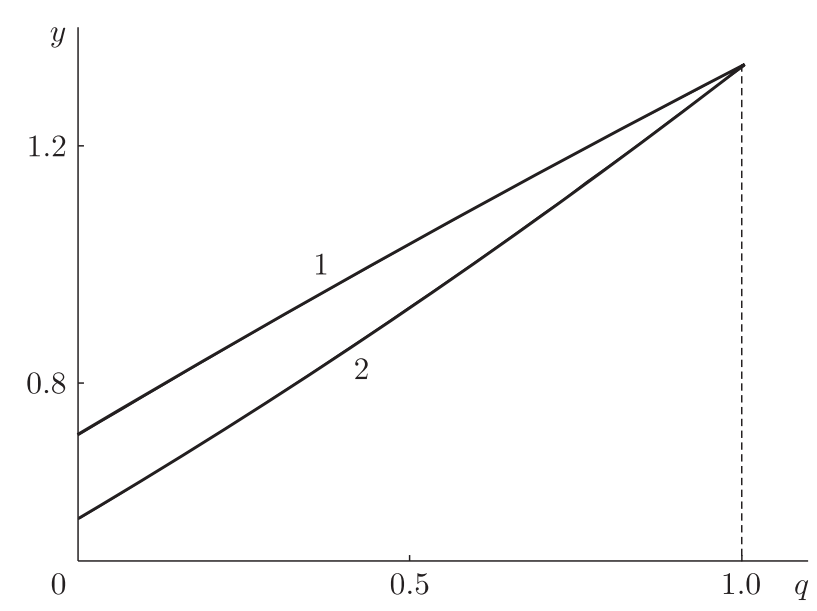

Рис. 2. Скачок температуры (кривая 1) и отклонение электронной температуры от температуры поверхности на границе металла (кривая 2)

Подставим в (39) разложение (12). Заметим, что

$$
\begin{aligned}
& \frac{g_{0}(\alpha)}{8 \pi \Delta_{0}(\alpha)} \int \varphi(x, \mu, c) c^{2} g(c) d^{3} c=\frac{g_{0}(\alpha) g_{2}(\alpha)}{\Delta_{0}(\alpha)} \cdot \frac{1}{2} \int_{-1}^{1} h_{1}(x, \mu) d \mu+\frac{1}{2} \int_{-1}^{1} h_{2}(x, \mu) d \mu, \\
& -\frac{g_{2}(\alpha)}{8 \pi \Delta_{0}(\alpha)} \int \varphi(x, \mu, c) g(c) d^{3} c=-\frac{g_{0}(\alpha) g_{2}(\alpha)}{\Delta_{0}(\alpha)} \cdot \frac{1}{2} \int_{-1}^{1} h_{1}(x, \mu) d \mu .
\end{aligned}
$$

Складывая два последних равенства, получаем профиль распределения температуры:

$$
\frac{\delta T(x)}{T_{\mathrm{s}}}=\frac{1}{2} \int_{-1}^{1} h_{2}(x, \mu) d \mu .
$$

Подставим в (40) равенства (17) и (22) и найдем явное выражение профиля температуры:

$$
\frac{\delta T(x)}{T_{\mathrm{s}}}=\varepsilon_{T}+g_{T}^{+} x+\frac{1}{2 \pi} \int_{-\infty}^{\infty} e^{i k x} E(k) d k .
$$

С учетом четности функции $E(k)$, а также равенства (30) из (41) получаем

$$
\frac{1}{g_{T}^{+}} \frac{\delta T(x)}{T_{\mathrm{s}}}=C_{T}(q)+x+\frac{1+q}{\pi} \int_{0}^{\infty} \cos (k x)\left[\sum_{n=0}^{\infty}(1-q)^{n} E_{n}(k)\right] d k .
$$

Как видно из приведенных выражений величины $C_{T}(q)$ и $\delta T(x)$ расходятся при стремлении коэффициента зеркальности $q$ к единице. Поэтому для анализа их поведения удобно ввести величины

$$
\begin{aligned}
y_{1}(q) & =(1-q) C_{T}(q), \\
y_{2}(q, x) & =(1-q)\left\{C_{T}(q)+\frac{1+q}{\pi} \int_{0}^{\infty} \cos (k x)\left[\sum_{n=0}^{\infty}(1-q)^{n} E_{n}(k)\right] d k\right\} .
\end{aligned}
$$

Эти величины остаются конечными в указанном пределе. 
На рис. 2 приведены зависимости $y_{1}(q)$ (кривая 1$)$ и $y_{2}(q, 0)$ (кривая 2). По мере роста коэффициента зеркальности графики этих функций сближаются. При этом профиль температуры вблизи поверхности приближается к асимптотическому.

\section{7. ЗАКЛЮЧЕНИЕ}

Используемое в работе тау-приближение совпадает для задач электропроводности металла с тау-приближением с постоянной частотой столкновений электронов. Это приближение широко используется при анализе проводимости металла в различных ситуациях (например, для проволок), при изучении скин-эффекта и т.д. Оно не учитывает ряд тонких эффектов: увлечение фононов, отклонение от закона Видемана-Франка.

Рассматриваемая в работе задача носит имя Смолуховского, ибо в нейтральных газах проблема распределения температуры вблизи поверхности впервые рассматривалась Смолуховским в конце ХІХ столетия [10].

В настоящей работе получено аналитическое выражение для распределения (профиля) температуры в полупространстве металла (формула (42)) и вычислена величина скачка температуры при наличии нормального к поверхности металла потока тепла. Оказалось, что коэффициент скачка температуры не зависит от химического потенциала, а зависит лишь от величины коэффициента зеркальности $q$, т.е. определяется характером рассеяния электронов на поверхности металла.

Отметим, что при $q=0$ зеркально-диффузные граничные условия сводятся к чисто диффузным. Точное решение задачи с диффузными граничными условиями известно [1]. В работе [1] получено, что $\varepsilon_{T}(0)=0.71045 . \quad$ В настоящей работе в нулевом приближении получено, что $\varepsilon_{0}=0.66667$. Следовательно, относительная ошибка в нулевом приближении составляет 10.7\%. В первом приближении получаем $\varepsilon_{T}^{(1)}(0)=\varepsilon_{0}+\varepsilon_{1}=0.71285$. Относительная ошибка в первом приближении равна $0.33 \%$. Во втором приближении согласно (37) находим: $\varepsilon_{T}^{(2)}(0)=\varepsilon_{0}+\varepsilon_{1}+\varepsilon_{2}=0.71030$. Относительная ошибка во втором приближении равна $-0.02 \%$.

Таким образом, предлагаемый метод чрезвычайно эффективен и обладает быстрой сходимостью.

Из формулы (31) видно, что с ростом коэффициента зеркальности $q$ коэффициент скачка температуры монотонно растет, и, следовательно, согласно (42) профиль распределения температуры сдвигается вверх. Это объясняется тем, что по мере роста степени зеркальности отражения электронов от поверхности она становится все менее проницаемой для потока тепла.

\section{Список литературы}

[1] А. В. Латышев, А. А. Юшканов, ЖТФ, 74:11 (2004), 1-7.

[2] А. В. Латышев, А. А. Юшканов, ТМФ, 142:1 (2005), 93-111.

[3] К. Черчиньяни, Теория и приложения уравнения Больимана, Мир, М., 1978. 
[4] А. В. Латышев, ПММ, 54:4 (1990), 581-586.

[5] А. В. Латышев, А. А. Юшканов, ЖЭТФ, 114:3 (1998), 956-971.

[6] Л. Д. Ландау, Е. М. Лифшиц, Теоретическая физика, Т. 10, Физическая кинетика, Наука, М., 1979.

[7] А.А. Абрикосов, Введение в теорию нормальных металлов, Наука, М., 1972.

[8] А. В. Латышев, А. А. Юшканов, ТМФ, 134:2 (2003), 310-324.

[9] J. M. Keller, R. Fuchs, K. L. Kliewer, Phys. Rev. B, 12:6 (1975), 2012-2029.

[10] M. Smoluchowski, Wiedemann Ann., 64 (1897), 101-130; Ann. Phys., 300:1 (1898), $101-130$. 